\title{
A retrospective outcome study in the elder patient with locally advanced rectal cancer treated with hypofractionated or conventional preoperative radiotherapy
}

\author{
Florence Guillerme ${ }^{1}$, Jean Emmanuel Kurtz ${ }^{2}$, J ean Baptiste Clavier ${ }^{1}$, Catherine Schumacher ${ }^{1}$, \\ Cecile Brigand $^{3}$, Georges Noël ${ }^{1}$
}

1. Radiotherapy Department, Centre de lutte contre le cancer Paul Strauss, Strasbourg, France. 2. Oncology Department, Hôpitaux Universitaires de Strasbourg, France. 3. Hôpitaux Universitaires de Strasbourg, Pôle des pathologies digestives, France

Correspondence: Georges Noël. Address: Radiotherapy Department, CLCC Paul Strauss, 3 rue de la Porte de I'hôpital, BP 43, 67065 Strasbourg cedex, France. E-mail: gnoel@strasbourg.unicancer.fr

Received: December 30, 2012

Accepted: January 27, 2013

Online Published: February 16, 2013

DOI : $10.5430 /$ jst.v3n2p25

URL: http://dx.doi.org/10.5430/jst.v3n2p25

\section{Abstract}

Background: Neoadjuvant chemoradiotherapy is considered the standard approach for T3-4 M0 rectal cancer; however, the optimal dose remains undefined for the elderly. We performed a retrospective analysis to compare conventional (C) and hypofractionated (HF) schedules in elderly patients. We compared survival rates, local control and morbidity.

Methods: From 2000 to 2008, 177 patients older than 65 years with T3-4 M0 rectal cancer received preoperative radiotherapy according to either a conventional protocol (45 to $50.4 \mathrm{~Gy}$ in 1.8-2 Gy daily fractions) or a hypofractionated (39 Gy in 3 Gy daily fractions) protocol. Fifty-five patients in the conventional group and none of the patients in the hypofractionated group received concomitant chemotherapy. Both groups were equivalent in terms of their characteristics. The median follow-up was 36 months.

Results: The occurrence of early grade 3-4 radiation toxicity was equivalent between the 2 groups (7\%). Surgery was performed in $98 \%$ of the patients in the HF group versus $92 \%$ in the conventional $(p=0.08)$. The delay between radiotherapy and surgery was 22 days in the HF group versus 45 days in the conventional group ( $p=0.0021)$. The downstaging rates were $39 \%$ in HF group and $45 \%$ in the $\mathrm{C}$ group $(p=0.53$ ). For lower rectum tumors, the conservative surgery rates were $43 \%$ in the $\mathrm{HF}$ group and $35 \%$ in the $\mathrm{C}$ group, $(p=0.52)$. The postoperative death rates at 30 days were equal between the two groups (3\%). The 5 -year local control rates was $87.3 \%$ in group $\mathrm{C}$ and $91.7 \%$ in group $\mathrm{HF}(p=0.5)$. Based on a Kaplan-Meier analysis, the 1-, 3- and 5-year overall survival rates were $88 \%, 67 \%$ and $45 \%$, respectively, in the $\mathrm{C}$ group and $84 \%, 60 \%$ and $39 \%$, respectively, in the HF group $(p=0.28)$. In a multivariate analysis, the prognostic factors for overall survival were a Charlson index $<2(p=0.0034 \mathrm{HR}=0.3)$, $\mathrm{pT}$ stage $\leqslant 2(p=0.0042 \mathrm{HR}=0.16)$, pN0 stage ( $p=0.0072 \mathrm{HR}=0.388)$, and downstaging $(p=0.0498 \mathrm{HR}=0.651)$. Radiation schedule and concomitant chemotherapy had no impact.

Conclusion: In this series, the local control rates and the overall survival results are equivalent for patients treated with HP and $\mathrm{C}$ radiation schedules. As hypofractionated radiotherapy is more convenient for elderly patients and has equivalent morbidity, additional prospective studies with this population could be of great interest.

\section{Key words}

Rectal cancer, Radiotherapy, Elderly, Dose fractionation, Hypofractionated 


\section{I ntroduction}

The management of rectal cancer conventionally uses three modalities: radiotherapy, chemotherapy and surgery. For the last several years, the role of preoperative radiotherapy has been clearly established through randomized studies and meta-analyses ${ }^{[1,2]}$. The association of radiotherapy and chemotherapy has been studied in randomized trials. Chemotherapy is conventionally performed with 5 -fluorouracil (5FU) or an equivalent treatment. The addition of platins, such as oxaliplatin, is more recent and has produced potentially interesting results ${ }^{[3]}$. The combination of radiotherapy and chemotherapy before surgery provides important benefits and has improved the results of radiotherapy alone ${ }^{[4]}$. The radiation dose delivered is conventionally between 45 and 50.4 Gy with 1.8 to 2 Gy daily fractions and 5 fractions per week. Hypofractionated schedule, with $25 \mathrm{~Gy}$ in five consecutive fractions of $5 \mathrm{~Gy}$, has been used with comparable results as conventional treatment but with probably more late complications. A more effective fractionation has not been clearly established. Although recent studies similar results in groups receiving two different fractionation protocols, the optimal regiment is still not established ${ }^{[5]}$.

The benefit of preoperative radiotherapy has been demonstrated in the elderly ${ }^{[1,2]}$ however, its use decreases with age because it has been suggested that treatment is less well tolerated in this population ${ }^{[6,7]}$. Furthermore, fatigability due to displacement should be taken into consideration when determining the number of fractions to be given to elderly patients. Here, we present the results of two schedules, 39 Gy in 13 fractions of 3 Gy and 45 Gy in 25 fractions of 1.8 Gy in order to do what to compare overall, survival, local control and downstaging rates, and to analyze morbidity between the two regiments of treatment.

Table 1. Patient characteristics

\begin{tabular}{|c|c|c|c|c|c|c|c|c|}
\hline & & $\begin{array}{l}\text { Total } \\
\mathbf{N}=177\end{array}$ & $\%$ & C & $\%$ & HF & $\%$ & $\boldsymbol{P}$ \\
\hline \multirow{2}{*}{ Age } & $<75$ & 97 & $55 \%$ & 61 & $59 \%$ & 36 & $49 \%$ & \multirow{2}{*}{0.2} \\
\hline & $\geq 75$ & 80 & $45 \%$ & 43 & $41 \%$ & 37 & $51 \%$ & \\
\hline \multirow{2}{*}{ Gender } & Male & 110 & $62 \%$ & 59 & $57 \%$ & 51 & $70 \%$ & \multirow{2}{*}{0.08} \\
\hline & Female & 67 & $38 \%$ & 45 & $43 \%$ & 22 & $30 \%$ & \\
\hline \multicolumn{2}{|l|}{ BMI median } & \multicolumn{2}{|c|}{$25.7(18.5-41.3)$} & \multicolumn{2}{|c|}{$25.9(19.6-41.3)$} & \multicolumn{2}{|c|}{$25.6(18.5-36.2)$} & NS \\
\hline \multirow{2}{*}{ PS WHO } & 0 & 106 & $60 \%$ & 61 & $59 \%$ & 45 & $61 \%$ & \multirow{2}{*}{0.75} \\
\hline & $\geq 1$ & 70 & $40 \%$ & 42 & $41 \%$ & 28 & $38 \%$ & \\
\hline \multirow{2}{*}{ Charlson Score } & $0-2$ & 151 & $86 \%$ & 89 & $86 \%$ & 62 & $85 \%$ & \multirow{2}{*}{0.82} \\
\hline & $>2$ & 25 & $14 \%$ & 14 & $14 \%$ & 11 & $15 \%$ & \\
\hline \multirow{2}{*}{ Tumor Location } & Mid/High & 72 & $41 \%$ & 33 & $32 \%$ & 39 & $53 \%$ & \multirow{2}{*}{0.005} \\
\hline & Low & 105 & $59 \%$ & 71 & $68 \%$ & 34 & $47 \%$ & \\
\hline \multirow{2}{*}{$\mathrm{T}$} & $\mathrm{T} 3$ & 151 & $86 \%$ & 87 & $84 \%$ & 64 & $90 \%$ & \multirow{2}{*}{0.27} \\
\hline & $\mathrm{T} 4$ & 24 & $14 \%$ & 17 & $16 \%$ & 7 & $10 \%$ & \\
\hline \multirow{2}{*}{$\mathrm{N}$} & No & 81 & $63 \%$ & 46 & $56 \%$ & 35 & $74 \%$ & \multirow{2}{*}{0.04} \\
\hline & $\mathrm{N}+$ & 48 & $37 \%$ & 36 & $44 \%$ & 12 & $26 \%$ & \\
\hline Mucinous component & Yes & 16 & $9 \%$ & 9 & $9 \%$ & 7 & $9 \%$ & 1 \\
\hline \multirow{2}{*}{ CA 19.9} & Normal & 126 & $85 \%$ & 69 & $83 \%$ & 57 & $88 \%$ & \multirow{2}{*}{0.49} \\
\hline & Abnormal & 22 & $15 \%$ & 14 & $17 \%$ & 8 & $12 \%$ & \\
\hline \multirow{2}{*}{ CEA } & Normal & 110 & $70 \%$ & 59 & $63 \%$ & 51 & $78 \%$ & \multirow{2}{*}{0.053} \\
\hline & Abnormal & 48 & $30 \%$ & 34 & $37 \%$ & 14 & $21 \%$ & \\
\hline
\end{tabular}

N: Number of patients; C: Conventional; HF: Hypofractionated 


\section{Materials and methods}

\subsection{Patients}

We performed a retrospective analysis of patients over 65 years of age who were treated with preoperative radiotherapy for locally advanced rectal cancer between January 2000 and December 2008. All patients' characteristics are reported Table 1. All the tumors were T3-T4 with or without lymph node involvement. More patients $>$ N0 are in the group C than in the group HF, $44 \%$ and $26 \%$ respectively. Patients who were treated with exclusive radiochemotherapy or postoperative radiotherapy and patients with metastasis were excluded from the analysis. All the tumors were considered as resectable. The patients had received no previous treatment for the rectal tumor, except for a derivation colostomy.

Two radiotherapy schedules were used: the classical (C) schedule, which delivered a dose of 45-50 Gy in 1.8 to 2 Gy daily fractions with 5 fractions per week, and the hypofractionated schedule (HF), which delivered a dose of 39 Gy in 13 fractions of 3 Gy with 5 fractions weekly. A total of 177 consecutive patients who fulfilled the inclusion criteria were included in this study: 104 patients received the $\mathrm{C}$ schedule, and 73 received the HF schedule. For all the patients, the median age was 74 years (range: 65 to 87.6 years). In group $C$, the median age was 73.2 years (range: 65 to 87.6 years, mean 73.8 years), and in group HF, it was 75 (range: 65 to 86.8 years, mean 75.4 years) ( $p=0.053$ ). The study sample was comprised of $62 \%$ men and $38 \%$ women. The two groups were equivalent in terms of their general condition $(60 \%$ of the patients had a WHO PS score equal to 0$)$, their comorbidity score ( $86 \%$ of the patients had a Charlson score less than or equal to 2) and their median BMI at the time of diagnosis (25.7). Overall, the tumors were more frequently localized in the lower rectum (61\%), although there was a difference between the two groups: $68 \%$ of the tumors in group C were in the lower rectum compared to $47 \%$ for the group HF $(p=0.005)$. The pathology of the tumors was confirmed as adenocarcinoma in all the cases, with a mucinous component in $9 \%$ of the cases.

\subsection{Classification}

The TNM classification used was the UICC $2002^{[8]}$. The spread of each tumor was assessed through a locoregional clinical examination that included a rectal examination and imaging (rectal ultrasonography, CT or pelvic MRI). The staging included a thoraco-abdominal-pelvic CT or a chest radiograph and an abdominal ultrasound. In cases of disagreement between the clinical findings and the imaging results regarding the tumor stage, the worst stage was used. cT3-stage tumors were found in $84 \%$ of the patients in the $\mathrm{C}$ group and in $90 \%$ of the patients in the HF group $(p=0.27)$. There was a difference between the 2 groups in terms of the $\mathrm{N}$ stage: in the $\mathrm{C}$ group, $56 \%$ of patients were staged at $\mathrm{cN} 0$ compared to $74 \%$ in the HF group $(p=0.04)$. In our evaluation of biological tumor markers, we found that CEA was normal in $63 \%$ of the patients in group C and in $78 \%$ of the patients in group HF, or $70 \%$ overall. CA $19-9$ was normal in $83 \%$ of the group C patients and in $88 \%$ of the group HF patients, or $88 \%$ overall.

\subsection{Treatment}

In all the cases, the radiotherapy was a three-dimensional conformational radiotherapy using photons of $6 \mathrm{MV}$ or more produced by linear accelerators. For all patients the same field technique has been performed using three photons fields, two lateral fields left and right and one posterior. All fields were treated every day. The target volume of radiotherapy was the macroscopic tumor or GTV (Gross Tumor Volume) defined by the CT-scan and the increased size of the lymph nodes. Information provided by other imaging modalities (ultrasonography, MRI, TEP-FDG) was taken into account for the delineation of the target volume. The CTV (Clinical Target Volume) included the mesorectum, the presacral area, and the anal canal, depending on the location of the tumor. Volumes were equal for the both groups.

For chemotherapy, 55 patients (31\%) received concurrent chemoradiotherapy with 5FU; among them, 73\% received per os chemotherapy, and 59\% were given oxaliplatin. Only the patients following the conventional fractionation schedule had concurrent chemotherapy. Two patients of this group did not receive a combined treatment. More patients initially, $>$ N0 
received chemoradiation than N0 patients, $42 \%$ and $34 \%$ respectively. Thirty-one patients received adjuvant chemotherapy with 5FU, leucovorin and oxaliplatine, 18 patients in the group $\mathrm{C}$ and 13 patients in the group HF.

Surgery was performed in $95 \%$ of the patients: one patient died during concurrent radio chemotherapy, one died before surgery due to comorbidity, two had distant metastases detected after the combined therapy, and four died for unknown reasons. The median interval between the completion of radiotherapy and surgery was 41 days for the patients in groups $\mathrm{C}$ and 11 days for the patients in group HF.

\subsection{Follow-up}

The follow-up was continued until the date of death or as part of the study until December 2010. The overall median follow-up period was 35.7 months (1 to 118.9 months). It was 34.1 months (1.5 to 118.9) for the patients of group C and 39.1 months ( 1 to 118.4 months) for the patients of group HF ( $p=0.1$ ).

\section{Statistical analysis}

Chi-squared test or Fisher's exact test were used for the analysis of qualitative variables, and ANOVA or a t-test was performed for quantitative variables. The survival rates were calculated by a Kaplan-Meier analysis. The survival time was the period between the date of the first irradiation and the date of death or the date of the last follow-up. The period of survival without recurrence was the period between the date of the first irradiation and the date of the first relapse (local or metastatic), the date of death or the date of the last follow-up.

A univariate analysis was performed to identify prognostic factors [age, gender, tumor classification, schedule of radiotherapy, concomitant radiotherapy, adjuvant chemotherapy, chemotherapy drugs, time between RT and surgery, OMS performance status, Charlson score, BMI class, characteristics of pathology (margins, angioinvasions, adenopathy...)]. The variables that were considered significant in the univariate analysis $(p<0.05)$ were included in a multivariate analysis. The multivariate analysis of the prognostic factors was performed using the Cox regression model with proportional rates to calculate a hazard ratio (HR) and the $95 \%$ confidence interval (CI). All the tests were performed using the software StatView v5.0 (SAS Institute Inc., Cary, NC).

\section{Results}

A conventional fractionation protocol was administered in $59 \%$ of the cases (104 patients). For the patients in the $\mathrm{C}$ group, the median dose was $46.8 \mathrm{~Gy}$ (range: 23.4 to $56 \mathrm{~Gy}$, mean $47.4 \mathrm{~Gy}$ ) and the mean duration was 40 days ( $\mathrm{SE}=0.76$ ). For the patients in the HF group, the median dose was $39 \mathrm{~Gy}$ (range: 36-39 Gy, mean $38.9 \mathrm{~Gy}$ ) and the mean duration was 19.8 days ( $\mathrm{SE}=0.27$ ). The radiotherapy was completed as expected for $99 \%$ of the patients in the HF group and $96 \%$ of the patients in the $\mathrm{C}$ group $(p=0.65$ ). The treatment duration was more than $20 \%$ longer than the theoretical target for $16 \%$ of the patients in group $\mathrm{C}$ and $27 \%$ of the patients in group HF $(p=0.09)$.

\subsection{Chemotherapy}

The patients in the HF group did not receive concurrent chemotherapy. Among the patients in group C, 55 patients ( $52 \%$ of patients) received concurrent chemoradiation. The indications for chemotherapy included a younger age $(\mathrm{OR}=0.163$ $\mathrm{CI} 95 \%=0.045-0.590, p=0.0057)$ and higher a $\mathrm{pN}$ stage $(\mathrm{OR}=6.255 \mathrm{CI} 95 \%=2.080-18.816 ; p=0.0011)$.

\subsection{Surgery}

Overall, $95 \%$ of the patients underwent surgery, including $98 \%$ of the patients in group HF and $94 \%$ of the patients in group $\mathrm{C}(p=0.08)$. The mean delay between completion of radiotherapy and surgery were 22 and 45 days for group $\mathrm{HF}$ and group $\mathrm{C}$, respectively $(p=0.0021)$. A downstaging was obtained for $41 \%$ of the patients in group $\mathrm{HF}$ and $45 \%$ of the 
patients in group $\mathrm{C}(p=0.74)$. According to the multivariate analysis, a delay between the end of radiotherapy and surgery more than 6 weeks $(\mathrm{OR}=3 \mathrm{CI} 95 \%=1.341-7.662, p=0.0088)$ and a T4 stage $(\mathrm{OR}=0.27 \mathrm{CI} 95 \%=0.086-0.825, p=0.0218)$ were favorable prognostic factors of downstaging. The rates of complete pathological response (pT0) were $6.7 \%$ and $1.4 \%$ ( $p=0.14$ ) for groups $\mathrm{C}$ and HF, respectively. For tumors of the lower third of the rectum, the sphincter preservation rates were $34 \%$ and $38 \%$ for groups $\mathrm{C}$ and $\mathrm{HF}$, respectively $(p=0.82)$ For the other locations, these rates were $66 \%$ and $81 \%$, respectively $(p=0.26)$.

Figure 1. Overall survival curves according to the radiotherapy schedule

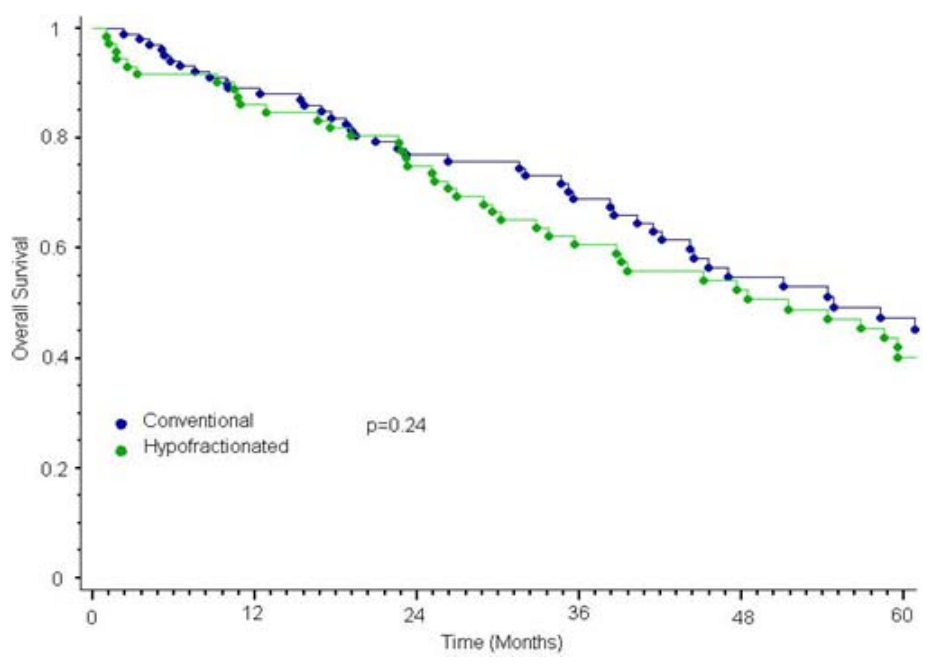

\subsection{Local control}

There was no difference of local or regional (nodes) control rates between the two groups. Median times of loco-regional control were not reached in the both groups. There were 9 locoregional relapses in the $\mathrm{C}$ group and 4 cases in the HF group. The 1-, 3- and 5-year local control rates were 96.5, 89.3 and 87.3\% in the $\mathrm{C}$ group and 98.3, 91.7 and $91.7 \%$ in the HF group. No prognostic factor was retrieved.

Figure 2. Disease-free survival curves according to the radiotherapy schedule

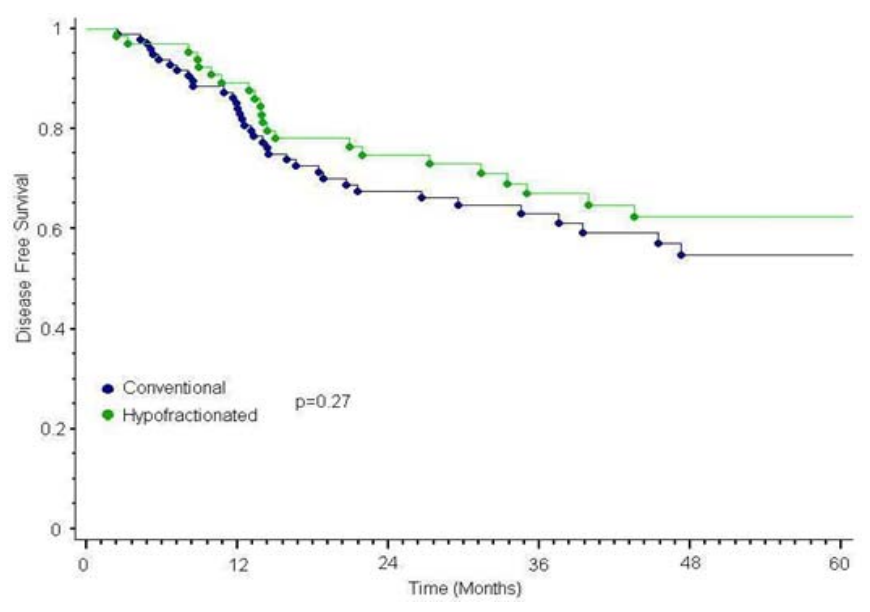

\subsection{Survival}

There was no difference in disease-free survival (DFS), and overall survival (OS), between the two schedules of radiotherapy, $p=0.73$ and $p=0.97$, respectively (Figures 1 and 2 ). 
Overall, the 1,3 and 5 -year OS rates were $86.7 \%, 64.4 \%$ and $41.9 \%$. For the $\mathrm{C}$ group, the rates were $88 \%, 67 \%$ and $45 \%$, and for the HF group, the rates were $84 \%, 60 \%, 39 \%$, respectively $(p=0.28)$ (Figure 1). In the multivariate analysis, preoperative favorable prognostic factors for OS was a Charlson score $<2$ (HR $=0.3$ CI95\% $=0.137-0.674, p=0.0034)$ Table 2). Postperative favorable prognostic factors for OS were pT stage $\leqslant 2(\mathrm{HR}=0.16 \mathrm{CI} 95 \%=0.045-0.561, p=0.0042)$, stage $\mathrm{pN} 0(\mathrm{HR}=0.388 \mathrm{CI} 95 \%=0.194-0.774, p=0.0072)$ and downstaging $(\mathrm{HR}=0.651 \mathrm{Cl} 95 \%=1.001-7.695 ; p=0.0498)$ (Table 3). There was no treatment-related predictive factor for DFS (Table 4).

Table 2. Preoperative predictive factors for overall and disease-free survival

\begin{tabular}{|c|c|c|c|c|c|c|c|c|c|}
\hline & & \multicolumn{4}{|c|}{ Overall Survival } & \multicolumn{4}{|c|}{ Disease-Free Survival } \\
\hline & & $\mathbf{N}$ & EVT & $P$ (Uni) & $P$ (Multi) & $\mathbf{N}$ & EVT & $P$ (Uni) & $P$ (Multi) \\
\hline \multirow{2}{*}{ Age } & $<75$ & 97 & 46 & \multirow{2}{*}{0.034} & \multirow{2}{*}{ NS } & 95 & 35 & \multirow{2}{*}{0.73} & \multirow{2}{*}{ NS } \\
\hline & $\geq 75$ & 80 & 46 & & & 78 & 24 & & \\
\hline \multirow{2}{*}{ PS WHO } & 0 & 106 & 56 & \multirow{2}{*}{0.041} & \multirow{2}{*}{ NS } & 105 & 37 & \multirow{2}{*}{0.58} & \multirow{2}{*}{ NS } \\
\hline & $\geq 1$ & 70 & 36 & & & 67 & 22 & & \\
\hline \multirow{2}{*}{ Charlson Score } & $0-2$ & 151 & 73 & \multirow{2}{*}{0.0005} & \multirow{2}{*}{0.0034} & 149 & 51 & \multirow{2}{*}{0.42} & \multirow{2}{*}{ NS } \\
\hline & $>2$ & 25 & 19 & & & 23 & 8 & & \\
\hline \multirow{2}{*}{$\mathrm{T}$} & 3 & 151 & 78 & \multirow{2}{*}{0.5} & \multirow{2}{*}{ NS } & 148 & 47 & \multirow{2}{*}{0.033} & \multirow{2}{*}{ NS } \\
\hline & 4 & 24 & 13 & & & 23 & 11 & & \\
\hline \multirow{2}{*}{$\mathrm{N}$} & 0 & 81 & 32 & \multirow{2}{*}{0.084} & \multirow{2}{*}{ NS } & 80 & 15 & \multirow{2}{*}{$<0.0001$} & \multirow{2}{*}{ NS } \\
\hline & $1-2$ & 48 & 27 & & & 47 & 26 & & \\
\hline \multirow{2}{*}{ Location } & Mid/high & 72 & 39 & \multirow{2}{*}{0.64} & \multirow{2}{*}{ NS } & 71 & 20 & \multirow{2}{*}{0.12} & \multirow{2}{*}{ NS } \\
\hline & Low & 105 & 53 & & & 102 & 39 & & \\
\hline \multirow{2}{*}{$\begin{array}{l}\text { Mucinous } \\
\text { component }\end{array}$} & Yes & 16 & 11 & \multirow{2}{*}{0.51} & NS & 16 & 7 & 044 & NS \\
\hline & No & 161 & 81 & & NS & 157 & 57 & 0.44 & NS \\
\hline CA 199 & Normal & 126 & 65 & 03 & NS & 123 & 43 & 0025 & NS \\
\hline & Abnormal & 22 & 16 & & & 22 & 12 & & \\
\hline 0 & $<10 \%$ & 116 & 60 & 056 & NS & 115 & 42 & 08 & NS \\
\hline \% weight loss & $>10 \%$ & 22 & 9 & 0.56 & NS & 21 & 6 & 0.8 & NS \\
\hline
\end{tabular}

N: Number of patients; EVT: Number of events, U: Univariate analysis; M: Multivariate analysis; NS: no significant; RT: Radiotherapy; CT: Chemotherapy

Table 3. Postoperative predictive factors for overall and disease-free survival

\begin{tabular}{|c|c|c|c|c|c|c|c|c|c|}
\hline & & \multicolumn{4}{|c|}{ Overall Survival } & \multicolumn{4}{|c|}{ Disease-Free Survival } \\
\hline & & $\mathbf{N}$ & EVT & $P$ (Uni) & $P$ (Multi) & $\mathbf{N}$ & EVT & $P$ (Uni) & P (Multi) \\
\hline \multirow{2}{*}{$\mathrm{pT}$} & $0-2$ & 59 & 16 & \multirow{2}{*}{0.0004} & \multirow{2}{*}{0.0042} & 59 & 8 & \multirow{2}{*}{$<0.0001$} & \multirow{2}{*}{ NS } \\
\hline & $3-4$ & 102 & 63 & & & 100 & 46 & & \\
\hline \multirow{2}{*}{$\mathrm{pN}$} & 0 & 118 & 45 & \multirow{2}{*}{$<0.0001$} & \multirow{2}{*}{0.0072} & 117 & 28 & \multirow{2}{*}{$<0.0001$} & \multirow{2}{*}{ NS } \\
\hline & $1-2$ & 56 & 45 & & & 53 & 29 & & \\
\hline \multirow{2}{*}{ Angioinvasion } & Yes & 11 & 9 & \multirow{2}{*}{0.008} & \multirow{2}{*}{ NS } & 10 & 6 & \multirow{2}{*}{0.0036} & \multirow{2}{*}{0.036} \\
\hline & No & 88 & 41 & & & 87 & 24 & & \\
\hline \multirow{2}{*}{ Resection margin } & R0 & 149 & 74 & \multirow{2}{*}{0.41} & \multirow{2}{*}{ NS } & 147 & 49 & \multirow[b]{2}{*}{0.19} & \multirow{2}{*}{ NS } \\
\hline & $\mathrm{R} 1-\mathrm{R} 2$ & 9 & 6 & & & 9 & 5 & & \\
\hline \multirow{2}{*}{ Downstaging } & Yes & 69 & 22 & \multirow{2}{*}{0.0018} & \multirow{2}{*}{0.0498} & 69 & 13 & \multirow{2}{*}{0.0007} & \multirow{2}{*}{ NS } \\
\hline & No & 90 & 56 & & & 88 & 40 & & \\
\hline
\end{tabular}

N: Number of patients; EVT: Number of events, U: Univariate analysis; M: Multivariate analysis; NS: no significant; RT: Radiotherapy; CT: Chemotherapy 
For The DFS, 56 events have been observed. Overall, the 1, 3 and 5-year DFS rates were $84.5 \%, 64.0 \%$ and $57 \%$. For the $\mathrm{C}$ group, the rates were $82.4 \%, 63.1 \%$ and $54.7 \%$, and for the HF group, the rates were $87.7 \%, 65.7 \%, 60.3 \%$, respectively $(p=0.28)$ (Figure 2). There was no preoperative favorable prognostic factor for DFS (Table 2). The postoperative favorable prognostic factor for DFS was the absence of angio-invasions (HR $=0.181$ CI $95 \%=0.037-0.89, p=0.0356$ ) (Table 3 ). There was no treatment-related predictive factor for DFS (Table 4).

Table 4. Treatment-related predictive factors for overall and disease-free survival

\begin{tabular}{|c|c|c|c|c|c|c|c|c|c|}
\hline & & Ove & urviva & & & Dise & ree Sur & & \\
\hline & & $\mathbf{N}$ & EVT & $P$ (Uni) & $P$ (Multi) & $\mathbf{N}$ & EVT & $P$ (Uni) & $P$ (Multi) \\
\hline & yes & 166 & 84 & & & 163 & 55 & & \\
\hline Curative Surgery & & & & 0.0001 & NS & & & 0.08 & NS \\
\hline & no & 9 & 7 & & & 9 & 3 & & \\
\hline DT & $\begin{array}{l}<6 \\
\text { weeks }\end{array}$ & 114 & 66 & 023 & NSC & 112 & 39 & 00 & NS \\
\hline Ne surguly Dotay & $\begin{array}{l}\geq 6 \\
\text { weeks }\end{array}$ & 47 & 15 & & & 46 & 13 & & \\
\hline & RT & 101 & 56 & & & 99 & 30 & & \\
\hline Therapeutic schema & RTCT & 76 & 36 & 0.83 & NS & 74 & 29 & 0.13 & NS \\
\hline Fractionation & $\mathrm{C}$ & 104 & 45 & & & 102 & 37 & & \\
\hline schedule & $\mathrm{HF}$ & 73 & 47 & 0.24 & NS & 71 & 22 & 0.27 & NS \\
\hline Radiotherapy & $<20 \%$ & 140 & 74 & & & 137 & 48 & & \\
\hline lengthening & $>20 \%$ & 37 & 18 & 0.77 & NS & 36 & 11 & 0.9 & NS \\
\hline
\end{tabular}

N: Number of patients; EVT: Number of events, U: Univariate analysis; M: Multivariate analysis; NS: no significant RT: Radiotherapy; CT: Chemotherapy

\subsection{Toxicity}

There was no significant difference in the acute grade 3 or 4 toxicities between the 2 schedules (7\%). In the multivariate analysis, pretreatment loss of weight less than $10 \%$ was a favorable prognostic factor of toxicity (HR 0.914 CI95\% $=0.845-0.988, p=0.0243)$. Age, general condition, comorbidities, fractionation of radiotherapy and concomitant chemotherapy were not prognosticators. For either iatrogenic deaths (5\%) or postoperative mortality at 30 days (3\%) and 6 months $(8 \%)$, there was also no difference between the 2 regimens.

\section{Discussion}

The standard treatment of locally advanced rectal tumors combines preoperative radiotherapy and concurrent chemotherapy, followed by surgical resection ${ }^{[9]}$. Complete removal of the tumor remains a determinant of the survival of elderly patients. The benefit of preoperative radiotherapy has been demonstrated in the elderly ${ }^{[1,2]}$. A Swedish trial showed an improvement of local control irrespective of age ${ }^{[10]}$, and studies have shown that pelvic radiotherapy is clearly achievable in elderly patients ${ }^{[6]}$. However, its use decreases with age because it has been suggested that treatment is less well tolerated in this population ${ }^{[11]}$. 
In a retrospective study of 534 patients, Ayanian et al. ${ }^{[12]}$ showed that the rates of radiotherapy use, for patients aged $<55$, $55-74,75-85$ and $\geqslant 85$ year-old, were $81.7 \%$, approximately $70 \%$, less than $50 \%$ and $14.3 \%$, respectively. A combination of radiochemotherapy was prescribed more frequently for patients less than 55 years old than for older patients ${ }^{[12]}$. Similarly, a study by Jung et al. ${ }^{[7]}$ that included 15,104 patients showed a decrease in the use of radiotherapy from $67 \%$ for patients less than 75 years old to $34 \%$ for patients older than 75 years. Furthermore, radiotherapy use has been shown to improved quality of life ${ }^{[13]}$.

The question of dose and fractionation remains controversial. Hypofractionation has already been used for elderly with high efficiency and tolerance ${ }^{[14]}$. Two classical radiotherapy schedules, a protocol that delivers 45 to 50 Gy with conventional fractionation and the Swedish protocol consisting of 25 Gy given in five consecutive fractions, provided similar results in terms of local control. The short course was associated with a low rate of acute toxicity ${ }^{[15]}$, while the long course appeared to decrease the rate of late toxicity ${ }^{[9]}$. In the Stockholm I trial ${ }^{[16]}$, the monitoring data from patients who received 25 Gy in 5 fractions showed an increase in the occurrence of late complications. However, due to differences in the techniques used between our study and the previous investigation, the relationship between late complications and the radiotherapy protocol remains unclear. In our study, local control rates are comparable between the both groups. There was no difference of patient's characteristics between the two groups. It could be concluded that the HF treatment can be proposed to elderly. With only 13 locoregional relapses our results are equivalent to those obtained by others studies ${ }^{[9,15}$, ${ }^{16]}$. Furthermore, OS and DFS are comparable between both groups. In their study Bujko et al. compared the Swedish and the classical radiotherapy protocols in patients with locally advanced rectal tumors that were T3-T4 and resectable (patients mean age: 60 ). The grade 3 and 4 acute toxicity rates were higher in the chemoradiotherapy group (18.2 vs. 3.2\% $p<0.0001$ ), but the late severe toxicities rates were comparable. In terms of efficacy, OS and DFS at 4 years were not statistically different in the 2 groups $\left(67 \%\right.$ and $56 \%$, respectively) ${ }^{[17]}$. In our study, the rate of grade $\geqslant 3$ toxities remained low at $7 \%$. This could be explained by the low use of chemoradiation. However, the lonely prognostic factor of complication was loss of weight. If this factor may seem intuitively easy to understand, no study has found that data as a prognostic factor for complications. However, it is a data that is perhaps not systematically analyzed because it may initially seem irrelevant. In fact, weight loss is rare in patients with rectal cancer. Bujko et al showed that the tumor response was significantly improved by the combination chemoradiotherapy ( $16 \%$ vs. $1 \% p<0.0001)$, but the authors did not find significant differences in the rates of local recurrence, which were $9 \%$ for the short course and $14 \%$ for the classical course $(p=0.170)^{[17]}$. In our study, chemoradiation was not a prognostic factor neither for OS nor for DFS. However, the downstaging is slightly higher in the $\mathrm{C}$ group, but the reason is probably less in the fractionation of the treatment than in the time between end of radiotherapy and surgery, longer in the $\mathrm{C}$ group. This was in concordance with the results of the $90-01$ Lyon trial ${ }^{[18]}$. Compliance with the short course was $98 \%$ compared to $69 \%$ for the classical schedule. Radiochemotherapy has not increased the rate of sphincter preservation compared to the short schedule, with rates of $61 \%$ and $58 \%(p=0.57)$, respectively ${ }^{[19]}$. In our study, there was no difference of sphincter preservation, but the treatment was not performed for this goal. The same hypofractionated protocol (39 Gy in 13 fractions) has been previously used in the Lyon trial with this objective but failed to prove its superiority comparatively to le conventional schedule ${ }^{[18]}$. In practice, elderly patients are usually excluded from prospective trials ${ }^{[20]}$, and most studies that have analyzed the role of chemoradiation found a benefit in terms of local control and downstaging, but not overall survival ${ }^{[4,17,21-23]}$.

The possibility that hypofractionated radiotherapy protocols could eliminate the need for preoperative chemotherapy makes this type of schedule an attractive option in the elderly. In conclusion, this retrospective study showed that the use of hypofractionated irradiation for the treatment of rectal cancer in the elderly is well tolerated with no negative impacts in terms of overall survival or local control. Prospective studies should be performed to investigate the use of hypofractionation in this population because many questions remain about the optimal strategy.

\section{Conflict of interests}

The authors declare that they have no conflict of interests. 


\section{References}

[1] Anonymous. Adjuvant radiotherapy for rectal cancer: a systematic overview of 8,507 patients from 22 randomised trials. Lancet. 2001; 358: 1291-304. http://dx.doi.org/10.1016/S0140-6736(01)06409-1

[2] Camma, C., Giunta, M., Fiorica, F., Pagliaro, L., Craxi, A. and Cottone, M. Preoperative radiotherapy for resectable rectal cancer: A meta-analysis. JAMA. 2000; 284: 1008-15. PMid:10944647 http://dx.doi.org/10.1001/jama.284.8.1008

[3] Gerard, J.P., Azria, D., Gourgou-Bourgade, S., Martel-Laffay, I., Hennequin, C., Etienne, P.L., et al. Comparison of two neoadjuvant chemoradiotherapy regimens for locally advanced rectal cancer: results of the phase III trial ACCORD 12/0405-Prodige 2. J Clin Oncol. 2010; 28: 1638-44. PMid:20194850 http://dx.doi.org/10.1200/JCO.2009.25.8376

[4] Gerard, J.P., Conroy, T., Bonnetain, F., Bouche, O., Chapet, O., Closon-Dejardin, M.T., et al. Preoperative radiotherapy with or without concurrent fluorouracil and leucovorin in T3-4 rectal cancers: results of FFCD 9203. J Clin Oncol. 2006; 24: 4620-5. PMid:17008704 http://dx.doi.org/10.1200/JCO.2006.06.7629

[5] Gerard, J.P., Azria, D., Gourgou-Bourgade, S., Martel-Laffay, I., Hennequin, C., Etienne, P.L., et al. Comparison of two neoadjuvant chemoradiotherapy regimens for locally advanced rectal cancer: results of the phase III trial ACCORD 12/0405-Prodige 2. J Clin Oncol. 28: 1638-44. PMid:20194850 http://dx.doi.org/10.1200/JCO.2009.25.8376

[6] Pignon, T., Horiot, J.C., Bolla, M., van Poppel, H., Bartelink, H., Roelofsen, F., et al. Age is not a limiting factor for radical radiotherapy in pelvic malignancies. Radiother Oncol. 1997; 42: 107-20. http://dx.doi.org/10.1016/S0167-8140(96)01861-0

[7] Jung, B., Pahlman, L., Johansson, R. and Nilsson, E. Rectal cancer treatment and outcome in the elderly: an audit based on the Swedish Rectal Cancer Registry 1995-2004. BMC Cancer. 2009; 9: 68. PMid:19245701

http://dx.doi.org/10.1186/1471-2407-9-68

[8] Greene, F.L. AJCC cancer staging manual. 2002; 1: 80-84.

[9] Valentini, V., Aristei, C., Glimelius, B., Minsky, B.D., Beets-Tan, R., Borras, J.M., et al. Multidisciplinary Rectal Cancer Management: 2nd European Rectal Cancer Consensus Conference (EURECA-CC2). Radiother Oncol. 2009; 92: 148-63. PMid:19595467 http://dx.doi.org/10.1016/j.radonc.2009.06.027

[10] Anonymous. Improved survival with preoperative radiotherapy in resectable rectal cancer. Swedish Rectal Cancer Trial. N Engl J Med. 1997; 336: 980-7. PMid:9091798 http://dx.doi.org/10.1056/NEJM199704033361402

[11] Schrag, D., Gelfand, S.E., Bach, P.B., Guillem, J., Minsky, B.D. and Begg, C.B. Who gets adjuvant treatment for stage II and III rectal cancer? Insight from surveillance, epidemiology, and end results--Medicare. J Clin Oncol. 2001; 19: 3712-8. PMid:11533092

[12] Ayanian, J.Z., Zaslavsky, A.M., Fuchs, C.S., Guadagnoli, E., Creech, C.M., Cress, R.D., et al. Use of adjuvant chemotherapy and radiation therapy for colorectal cancer in a population-based cohort. J Clin Oncol. 2003; 21: 1293-300. PMid:12663717 http://dx.doi.org/10.1200/JCO.2003.06.178

[13] Bouvier, A.M., Jooste, V., Bonnetain, F., Cottet, V., Bizollon, M.H., Bernard, M.P., et al. Adjuvant treatments do not alter the quality of life in elderly patients with colorectal cancer: a population-based study. Cancer. 2008; 113: 879-86. PMid:18521903 http://dx.doi.org/10.1002/cncr.23629

[14] Bentzen, S.M., Agrawal, R.K., Aird, E.G., Barrett, J.M., Barrett-Lee, P.J., Bliss, J.M., et al. The UK Standardisation of Breast Radiotherapy (START) Trial B of radiotherapy hypofractionation for treatment of early breast cancer: a randomised trial. Lancet. 2008; 371: 1098-107. http://dx.doi.org/10.1016/S0140-6736(08)60348-7

[15] Martling, A., Holm, T., Johansson, H., Rutqvist, L.E. and Cedermark, B. The Stockholm II trial on preoperative radiotherapy in rectal carcinoma: long-term follow-up of a population-based study. Cancer. 2001; 92: 896-902. http://dx.doi.org/10.1002/1097-0142(20010815)92:4<896::AID-CNCR1398>3.0.CO;2-R

[16] Cedermark, B., Johansson, H., Rutqvist, L.E. and Wilking, N. The Stockholm I trial of preoperative short term radiotherapy in operable rectal carcinoma. A prospective randomized trial. Stockholm Colorectal Cancer Study Group. Cancer. 1995; 75: $2269-75$. http://dx.doi.org/10.1002/1097-0142(19950501)75:9<2269::AID-CNCR2820750913>3.0.CO;2-I

[17] Bujko, K., Nowacki, M.P., Nasierowska-Guttmejer, A., Michalski, W., Bebenek, M. and Kryj, M. Long-term results of a randomized trial comparing preoperative short-course radiotherapy with preoperative conventionally fractionated chemoradiation for rectal cancer. Br J Surg. 2006; 93: 1215-23. PMid:16983741 http://dx.doi.org/10.1002/bjs.5506

[18] Francois, Y., Nemoz, C.J., Baulieux, J., Vignal, J., Grandjean, J.P., Partensky, C., et al. Influence of the interval between preoperative radiation therapy and surgery on downstaging and on the rate of sphincter-sparing surgery for rectal cancer: the Lyon R90-01 randomized trial. J Clin Oncol. 1999; 17: 2396. PMid:10561302

[19] Bujko, K., Nowacki, M.P., Nasierowska-Guttmejer, A., Michalski, W., Bebenek, M., Pudelko, M., et al. Sphincter preservation following preoperative radiotherapy for rectal cancer: report of a randomised trial comparing short-term radiotherapy vs. 
conventionally fractionated radiochemotherapy. Radiother Oncol. 2004; 72: 15-24. PMid:15236870 http://dx.doi.org/10.1016/j.radonc.2003.12.006

[20] Lewis, J.H., Kilgore, M.L., Goldman, D.P., Trimble, E.L., Kaplan, R., Montello, M.J., et al. Participation of patients 65 years of age or older in cancer clinical trials. J Clin Oncol. 2003; 21: 1383-9. PMid:12663731 http://dx.doi.org/10.1200/JCO.2003.08.010

[21] Bosset, J.F., Collette, L., Calais, G., Mineur, L., Maingon, P., Radosevic-Jelic, L., et al. Chemotherapy with preoperative radiotherapy in rectal cancer. N Engl J Med. 2006; 355: 1114-23. PMid:16971718 http://dx.doi.org/10.1056/NEJMoa060829

[22] Pasetto, L.M., Friso, M.L., Pucciarelli, S., Basso, U., Falci, C., Bortolami, A., et al. Rectal cancer neoadjuvant treatment in elderly patients. Anticancer Res. 2006; 26: 3913-23. PMid:17094422

[23] Lorchel, F., Peignaux, K., Crehange, G., Bosset, M., Puyraveau, M., Mercier, M., et al. Preoperative radiotherapy in elderly patients with rectal cancer. Gastroenterol Clin Biol. 2007; 31: 436-41. http://dx.doi.org/10.1016/S0399-8320(07)89407-3 